\title{
Bericht des Fachkollegiums Psychologie der Deutschen Forschungsgemeinschaft (DFG) und der DFG-Geschäftsstelle Psychologie
}

\author{
Elmar Brähler, Anne Brüggemann, Joachim C. Brunstein, Michael Diehl, \\ Edgar Erdfelder, Clemens Kirschbaum, Ulman Lindenberger, Erich Schröger, \\ Sabine Sonnentag, Rolf Ulrich und Hannelore Weber
}

\begin{abstract}
Nachdem das Fachkollegium Psychologie in der Psychologischen Rundschau (2010, 61/3, 147-149) über die Neuregelungen der Publikationsverzeichnisse bei DFG-Anträgen informiert und diese kommentiert hatte, wollen wir in diesem Bericht die Arbeit des DFG-Fachkollegiums Psychologie etwas allgemeiner vorstellen und einige wichtige Statistiken der DFG-Förderung in den letzten Jahren berichten. Aus diesen Informationen und auch aus unseren Erfahrungen als Mitglieder des Fachkollegiums lassen sich Empfehlungen für die Beantragung von DFG-Fördermitteln und den Umgang mit Förder- bzw. Ablehnungsentscheidungen ableiten, die wir abschließend darstellen und begründen werden.
\end{abstract}

Die hier berichteten Zahlen wurden größtenteils dem Fachkollegienbericht 2011 der DFG entnommen, der von der Gruppe Informationsmanagement der DFG-Geschäftsstelle zusammengestellt wurde. Teilweise wurden von der DFG-Geschäftsstelle zusätzliche Informationen speziell für den vorliegenden Bericht zusammengestellt. Als Informationsquellen haben wir außerdem die Webseiten der DFG, die Satzung der DFG, diverse Merkblätter und unsere Erfahrung im Fachkollegiumsalltag verwendet.

Fachkollegien und Geschäftsstelle: Zusammenarbeit bei der Entscheidungsvorbereitung von Anträgen

Die DFG ist die zentrale Selbstverwaltungsorganisation der deutschen Wissenschaft. Eine ihrer Kernaufgaben be- 
steht in der wettbewerblichen Auswahl der besten Forschungsvorhaben von Wissenschaftlerinnen und Wissenschaftlern an Hochschulen und Forschungsinstituten sowie in deren Finanzierung. Die DFG widmet sich zudem der Herstellung der Chancengleichheit im Wissenschaftssystem, der Förderung des wissenschaftlichen Nachwuchses, der Zusammenarbeit in der Wissenschaft und dem Austausch mit Wirtschaft und Gesellschaft sowie der Beratung von Politik und Öffentlichkeit.

Bei der Umsetzung dieser Aufgaben - insbesondere bei der Vorbereitung von Förderungsentscheidungen arbeiten Fachkollegien und die Geschäftsstelle eng zusammen. Es gibt 48 Fachkollegien, darunter das Fachkollegium 110 „Psychologie“ mit den gewählten Mitgliedern Elmar Brähler, Joachim C. Brunstein, Michael Diehl, Edgar Erdfelder, Clemens Kirschbaum, Ulman Lindenberger, Erich Schröger (Sprecher), Sabine Sonnentag, Rolf Ulrich und Hannelore Weber (stellvertretende Sprecherin). Die Fachkollegien sind primär bei der Qualitätssicherung der Begutachtung und bei der Vorbereitung von Förderentscheidungen der DFG involviert. Sie geben u. a. Förderempfehlungen auf der Basis von (in der Regel zwei) Gutachten fachkundiger Expertinnen und Experten, die zuvor von der DFG-Geschäftsstelle eingeholt wurden. Dies betrifft Einzelanträge, also Anträge auf Sachbeihilfen und Forschungsstipendien, Anträge im Emmy NoetherProgramm und im Heisenberg-Programm sowie Reinhart Koselleck-Projekte. Darüber hinaus sind die Fachkollegien in allen Prüfungsgruppen beteiligt, d. h. in sogenannten koordinierten Förderprogrammen (Sonderforschungsbereiche, Schwerpunktprogramme, Graduiertenkollegs, Forschergruppen). Fachkollegien sind auch für die Entwicklung fachbezogener Empfehlungen (z. B. Initiativen für neue Schwerpunktprogramme) zuständig und beraten DFG-Gremien und die Geschäftsstelle in fachbezogenen Angelegenheiten (z.B. in Bezug auf Erfordernisse in der Publikationsförderung oder Standards in der Grundausstattung).

Die eigentliche Begutachtung von Anträgen wird fast immer außerhalb der Fachkollegien durch nationale und internationale Gutachter und Gutachterinnen durchgeführt. Für die Psychologie wurden im Zeitraum 2008-2010 insgesamt 1570 Begutachtungen durch nationale und 298 Begutachtungen durch internationale Gutachter und Gutachterinnen vorgenommen. Durch die Trennung zwischen Begutachtung und der Bewertung dieser Begutachtung erfüllen die Fachkollegien also eine ähnliche Funktion wie die Editoren bei Zeitschriften mit externem Begutachtungssystem und tragen so zur Qualitätssicherung bei. Diese Trennung befördert die Transparenz der Begutachtungs- und Entscheidungskriterien sowie die Prüfung und Sicherstellung der Kompetenz und Fairness der Gutachten. Zudem ermöglicht sie den kompetitiven Vergleich der Anträge, die zur Entscheidung anstehen. In einem System mit begrenzten Ressourcen - bei Zeitschriften ist dies die Zahl der zur Verfügung stehenden Druckseiten, bei der DFG die zur Verfügung stehenden Fördermittel - lassen sich die einzelnen Beiträge (bei Zeitschriften) bzw. Anträge (bei der DFG) nicht immer nur für sich genommen begutachten, sondern müssen auch im Vergleich bewertet werden.
Die Fachkollegien stehen in engem Kontakt mit der DFG-Geschäftsstelle. Für die Psychologie sind dies die verantwortlichen Ansprechpartnerinnen Dr. Anne Brüggemann (Programmdirektorin) und Dr. Helga WeyertsSchweda (Referentin). Sie sind mit ihrem Team u. a. Anlaufstellen für Antragsteller und Antragstellerinnen. Auch betreuen sie die Fachkollegien, führen die formale Prüfung der Anträge durch, suchen Gutachterinnen und Gutachter für die eingehenden Anträge, d.h. sie organisieren und moderieren den gesamten Begutachtungs- und Entscheidungsprozess. Sie sind darüber hinaus Ansprechpartner für alle Fragen, die im Laufe der Förderung eines Projektes auftreten.

Um ein möglichst sachgerechtes und faires Entscheidungsverfahren zu gewährleisten, organisiert die DFG die Begutachtung und Entscheidung über Anträge in einem gestuften System mit gegenseitiger Kontrolle. Dabei dienen die Empfehlungen des Fachkollegiums der Vorbereitung der Entscheidung über die Förderanträge, die wiederum von unabhängigen Gremien getroffen werden. Diese sind der Hauptausschuss (für Anträge auf Förderung im Normal- und Schwerpunktverfahren, im Emmy Noetherund im Heisenberg-Programm sowie im Rahmen der Bibliotheksförderung) oder weitere Bewilligungsausschüsse der DFG, wie der Bewilligungsausschuss für Graduiertenkollegs oder der Bewilligungsausschuss für Sonderforschungsbereiche.

\section{Einblick in die Arbeit der Fachkollegien}

Das Fachkollegium Psychologie trifft sich viermal pro Jahr in der Geschäftsstelle. Dort werden die Anträge und die zugehörigen Gutachten diskutiert. Pro Sitzung handelt es sich um ca. 50-60 Einzelanträge. In der Kollegiumssitzung wird jeder Antrag nebst Gutachten von zwei Berichterstattern bzw. Berichterstatterinnen vorgestellt, wobei jedes Mitglied Einblick in alle Anträge und alle Gutachten hat. Für jeden Antrag wird ein Entscheidungsvorschlag erarbeitet, d.h. Bewilligung oder Ablehnung. Im Falle eines Bewilligungsvorschlags wird ein detaillierter Vorschlag über die zu bewilligenden Mittel erstellt. Die abschließenden Empfehlungen zu Anträgen werden gemeinschaftlich getroffen. Die Mitglieder der Fachkollegien geben keine Information an anfragende Antragsteller in Bezug auf ihre Anträge. Hierfür stehen die Ansprechpartnerinnen der Geschäftsstelle zur Verfügung. Selbstverständlich wirken Mitglieder des Fachkollegiums nicht bei Antragsverfahren mit, in denen sie möglicherweise befangen sind (u.a. eigene Anträge, Anträge eng kooperierender Kollegen und Kolleginnen, Anträge auch nicht kooperierender Kolleginnen und Kollegen aus demselben Institut oder derselben Universität).

Anträge mit eindeutigen Empfehlungen der Fachgutachten und eilige Anträge können auch zwischen den Sitzungsterminen in einem schriftlichen Verfahren behandelt werden. Das Prozedere ist hierbei analog zum mündlichen Verfahren. Darüber hinaus nehmen einzelne Fachkollegiaten bzw. -kollegiatinnen an diversen Treffen bei der DFG 
teil (z.B. Tagung der Sprecher und Sprecherinnen der Fachkollegien) und wirken an Begutachtungen im Rahmen koordinierter Förderprogramme (Forschergruppen, Schwerpunktprogramme, Graduiertenkollegs, Sonderforschungsbereiche) oder internationaler Programme (z. B. „Open Research Area for the Social Sciences“) mit. Auch Begutachtungen und Stellungnahmen für thematisch benachbarte Fachkollegien der DFG spielen eine nicht zu vernachlässigende Rolle.

\section{Einige Zahlen für den Zeitraum 2002-2010}

Im Fachkollegium Psychologie wurden im Jahr 2010 insgesamt 330 Anträge behandelt. In dieser Zahl sind die Anträge in koordinierten Förderprogrammen, die in der Regel durch separate Prüfgruppen und unter Einbeziehung eines Mitglieds des Fachkollegiums begutachtet werden, nicht berücksichtigt. In den Jahren davor wurden im Fachkollegium Psychologie 374 (2009), 358 (2008), 342 (2007), 328 (2006), 304 (2005), 323 (2004), 291 (2003) bzw. 250 Anträge (2002) entschieden. In Abbildung 1 sind die Förderquoten (Prozentzahl der bewilligten relativ zu Gesamtzahl der gestellten Anträge) für Neuanträge und für Fortsetzungsanträge getrennt für das Fachkollegium Psychologie und alle DFG-Fachkollegien über die Zeit dargestellt.

Man erkennt, dass über die Jahre hinweg meist etwas mehr als $40 \%$ der Neuanträge bewilligt wurden; dies gilt sowohl für das Fachkollegium Psychologie als auch für die DFG insgesamt. Im Vergleich zu den Förderquoten, die man aus Wissenschaftsorganisationen anderer Länder oder aus internationalen Wissenschaftsorganisationen

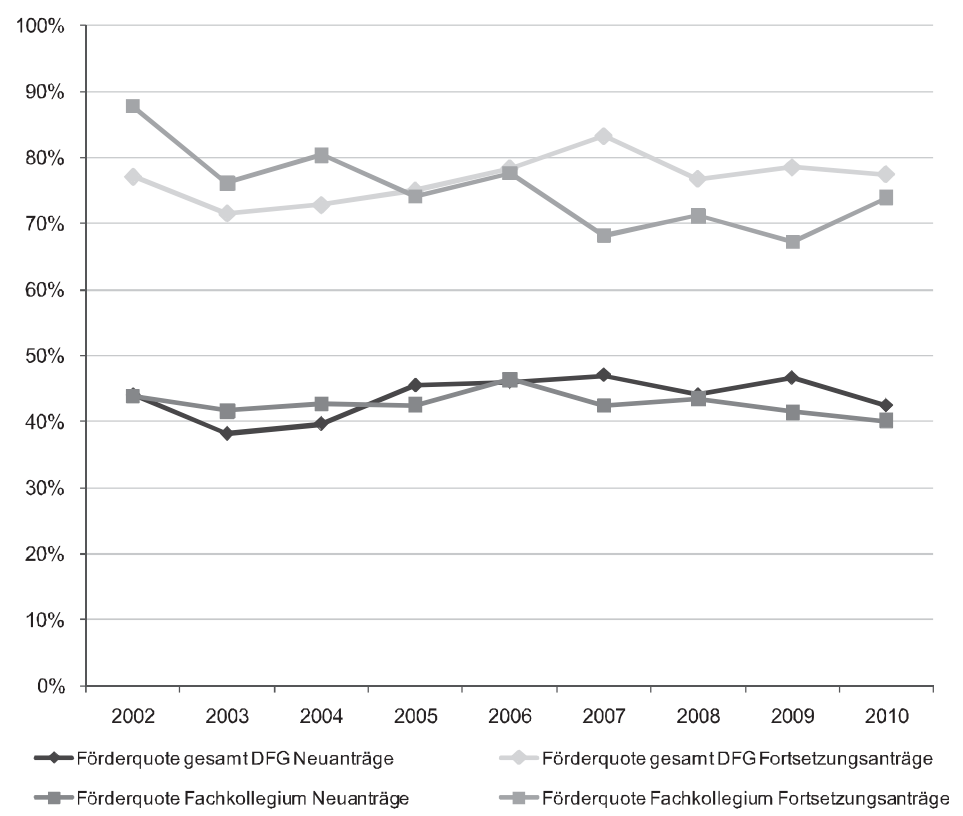

Abbildung 1. Entwicklung der Förderquoten des Fachkollegiums $110 \mathrm{im}$ Vergleich mit der Gesamtentwicklung: Neu- vs. Fortsetzungsanträge in der Einzelförderung 2002 bis 2010 (in \%); Quelle: DFG Fachkollegienbericht 2011. kennt (mit Förderquoten oft nur um die 10\%), sieht es für Antragsteller bei der DFG also günstig aus. Noch besser ist es um die Förderquoten von Fortsetzungsanträgen bestellt. Diese liegt über die Jahre (sowohl für die Psychologie als auch für die DFG insgesamt) stabil bei $70 \%$ und darüber. Die Quoten unterscheiden sich zwischen den Fachkollegien nicht sehr stark. Das bedeutet, dass es in der Psychologie ebenso schwer bzw. leicht ist ein DFGProjekt bewilligt zu bekommen wie etwa in den Sprachwissenschaften oder in der Biologie. Das gelegentlich geltend gemachte Argument, Anträge aus naturwissenschaftlichen Fächern würden strenger begutachtet und seltener bewilligt als Anträge aus den sozial- und geisteswissenschaftlichen Fächern (und seien daher, beispielsweise durch die Universitätsleitung, höher einzuschätzen) trifft also nicht zu.

Für Nachwuchswissenschaftler und Nachwuchswissenschaftlerinnen ist von besonderem Interesse, dass die Förderquoten für Erstanträge und Anträge auf eine eigene Stelle im Bereich der Förderquoten für alle Neuanträge liegen. Statistisch gibt es also weder eine Begünstigung des wissenschaftlichen Nachwuchses noch eine Begünstigung von etablierten Wissenschaftlerinnen und Wissenschaftlern. Letzteres ist eine häufig geäußerte Befürchtung, die in den vorliegenden Daten jedoch keine Bestätigung findet.

Bei abgelehnten Anträgen von Erstantragstellerinnen und Erstantragstellern ist das Fachkollegium in besonderem Maße bemüht, hilfreiche Rückmeldungen zu geben. Es kommt hier auch häufiger als in anderen Fällen vor, dass explizit eine Überarbeitungsempfehlung gegeben wird. Bei erfahrenen Antragstellern und Antragstellerinnen geht das Fachkollegium im Falle einer Ablehnung zumeist davon aus, dass die Rückmeldung der Gutachten ausreichend ist, um angemessen mit der Ablehnung umgehen zu können, also z. B. die Erfolgsaussichten einer Überarbeitung und Wiedereinreichung einschätzen zu können.

Es gibt keine speziellen Kriterien bei der Beurteilung von DFG-Erstanträgen und Anträgen auf eigene Stelle. Selbstverständlich wird der Bewertungsmaßstab in Bezug auf wissenschaftliche Vorleistungen (z. B. Publikationen) an die Karrierephase angepasst. Wie immer ist jedoch auch bei Erstanträgen und Anträgen auf eine eigene Stelle die wissenschaftliche Qualität des Antrags letztlich entscheidend.

Die Förderquote bei Forschungsstipendien liegt seit vielen Jahren deutlich über den Quoten der anderen Programme (z.B. bei $86 \%$ in 2010, $78 \%$ in $2009,60 \%$ in $2008,68 \%$ in 2007). Bei diesen Anträgen spielt der Qualifikationsaspekt eine sehr große Rolle. Darüber hinaus sind die Fördersummen bei Forschungsstipendien relativ gering und das „Preis-Leistungs-Verhältnis“ ist ausgesprochen günstig. Diese Faktoren können die hohen Bewilligungsquoten erklären.

Im Fall einer Projektbewilligung wird selbstverständlich nicht immer die volle Antragssumme be- 


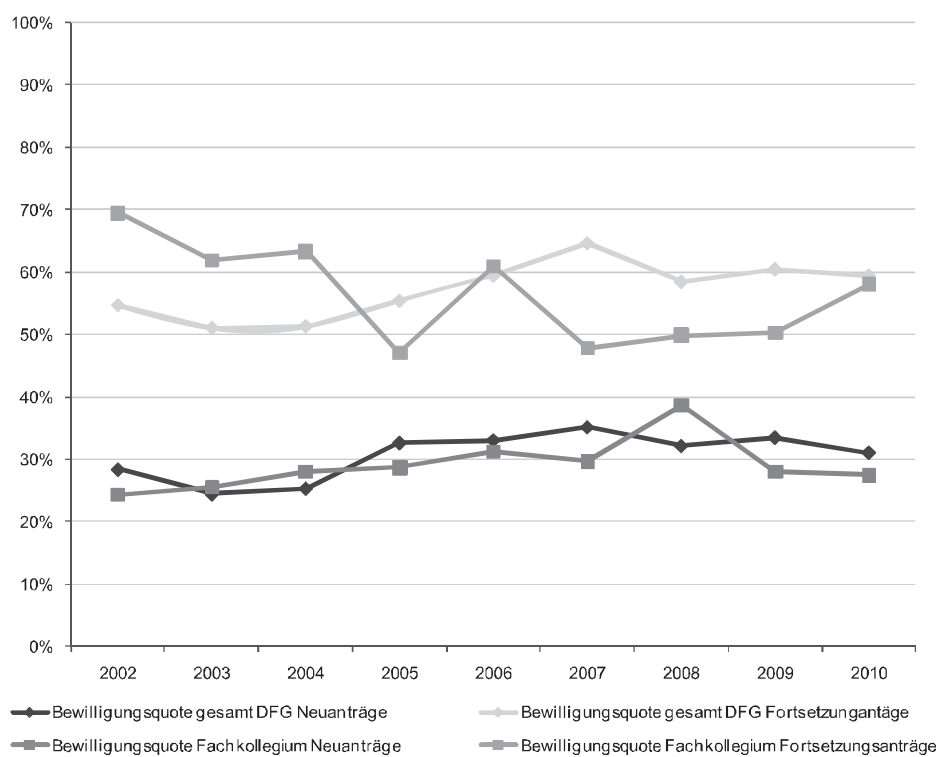

Abbildung 2. Entwicklung der Bewilligungsquoten des Fachkollegiums 110 im Vergleich mit der Gesamtentwicklung: Neu- vs. Fortsetzungsanträge in der Einzelförderung 2002 bis 2010 (in \%); Quelle: DFG Fachkollegienbericht 2011.

willigt. In Abbildung 2 sind die Bewilligungsquoten (Prozentzahl der bewilligten relativ zur Anzahl der beantragten Mittel) über die Jahre dargestellt, wiederum separat für die Psychologie und die DFG insgesamt.

Da sich die in Abbildung 2 eingehenden Zahlen auf die insgesamt beantragten Mittel beziehen - dort fließen somit auch die abgelehnten Anträge ein - lässt sich aus der Abbildung erschließen, dass de facto bei den bewilligten Projekten ein Großteil der beantragten Mittel bewilligt wird. Dies trifft wiederum für die Erst- und die Verlängerungsanträge und sowohl für das Fachkollegium Psychologie als auch für die DFG insgesamt zu. Wie Abbildung 2 entnommen werden kann, ist die Bewilligungsquote über die Jahre hinweg relativ konstant.

Wenn Kürzungen vorgenommen werden, so betreffen sie oftmals die Laufzeit der Projekte für den ersten Projektzeitraum (z. B. wenn das Projekt nur für zwei statt der beantragten drei Jahre bewilligt wird), aber auch die personelle Ausstattung (z. B. wenn eine Reduktion der Mittel für studentische Hilfskräfte angemessen erscheint) oder andere Positionen (z. B. werden Reisemittel für Kongresse pauschaliert). Zu beachten ist, dass Kürzungen für sich genommen nichts über die Erfolgsaussichten von möglichen Nachfolgeanträgen aussagen.

Insgesamt wurden 2010 für die Psychologie 69,9 Millionen Euro beantragt und 21,4 Millionen Euro bewilligt. In den Vorjahren betrugen die bewilligten Fördersummen (in der Reihenfolge 2009-2002) 21,6, 28,1, 20,3, 18,3, 13,7, 16,2, 14,7 bzw. 11,0 Millionen Euro. Die Mittel haben also in den letzten Jahren in der Psychologie beträchtlich zugenommen. Diese Zunahme ist durch eine Erhöhung des Antragsaufkommens in der Psychologie relativ zu manchen anderen Fachkollegien und durch eine Zunahme der Mittelzuweisung an die DFG insgesamt zu erklären. Die ge- nannten Bewilligungssummen beziehen sich wohlgemerkt nur auf die Einzelförderung (Einzelanträge, Forschungsstipendien, Emmy-Noether und Heisenberg-Programm und Reinhart-Koselleck-Projekte). Hinzu kommen Bewilligungen von Forschergruppen, Schwerpunktprogrammen, Graduiertenkollegs und in Sonderforschungsbereichen (im Jahr 2010 z.B. insgesamt weitere $10 \mathrm{Mio} €$ ). Aufgrund des verstärkten Wettbewerbs kann leider nicht ausgeschlossenen werden, dass es perspektivisch zu sinkenden Bewilligungsquoten kommt. Dies sollte jedoch nicht voreilig auf Budgetkürzungen attribuiert werden. Es ist wichtig, sich zu vergegenwärtigen, dass sich die vom Fachkollegium Psychologie zur Bewilligung empfohlenen Fördermittel in der Einzelförderung innerhalb der letzten 10 Jahre in etwa verdoppelt haben.

Eine Ausnahme bilden leider die Sonderforschungsbereiche. Aus Sonderforschungsbereichen flossen in den letzten Jahren weniger als eine Million Euro an Projekte aus der Psychologie. Diesbezüglich ist in der Psychologie also ein Defizit zu konstatieren. Dies wird auch klar, wenn man sich die absoluten Zahlen in der DFG ansieht: In 2010 wurden 456 Millionen Euro für Sonderforschungsbereiche bewilligt (davon 0,5 Millionen für psychologische Projekte) und 760 Millionen Euro für Einzelprojekte. Das Bewilligungsverhältnis Sonderforschungsbereiche vs. Einzelprojekte beträgt also bei der DFG insgesamt 0,6, für die Psychologie hingegen nur 0,02. Ein Grund für die unbefriedigende Präsenz der Psychologie in Sonderforschungsbereichen ist sicherlich, dass die Psychologie als relativ kleines Fach nur an wenigen Standorten stark genug ist, um federführend einen Sonderforschungsbereich (SFB) zu initiieren. Trotzdem sollte langfristig angestrebt werden, dass mehr (erfolgreiche) SFB-Initiativen aus der Psychologie kommen, ggf. in Verbindung mit Nachbardisziplinen.

Die Bewilligungsquoten sind für Männer und Frauen in der Psychologie relativ ähnlich (im Zeitraum 2008-2010 Männer 46,8 \% bzw. Frauen 45,0\%; 2005-2007: 49,6 bzw. 48,6). Dies trifft jedoch nicht für die Zahl der Anträge insgesamt zu: Es stellen mehr Männer als Frauen Anträge. In der Psychologie kommen derzeit etwa 35\% aller Anträge von Frauen, in der DFG insgesamt sind es etwa 19\% (Zeitraum 2008-2010). Im Zeitraum 2002-2004 kamen in der Psychologie etwa 27\% aller Anträge von Frauen, in der DFG insgesamt etwa 16\%. Obwohl der Anteil der Antragstellerinnen somit leicht gestiegen ist, bleibt festzuhalten, dass Frauen nach wie vor deutlich weniger Anträge als Männer stellen. Dies ist vermutlich in erster Linie dadurch bedingt, dass der Anteil der antragsberechtigten Frauen kleiner ist als der der Männer. Die Bewilligungschancen gestellter Anträge unterscheiden sich zwischen Männern und Frauen kaum.

\section{Empfehlungen für die Antragstellung}

Die im internationalen Vergleich relativ günstigen Förderquoten sollten Wissenschaftlerinnen und Wissenschaft- 
ler ermutigen, bei der DFG Anträge zu stellen. Dabei gilt: „Antragsberechtigt ist grundsätzlich jede Wissenschaftlerin und jeder Wissenschaftler in der Bundesrepublik Deutschland oder an einer deutschen Forschungseinrichtung im Ausland, deren wissenschaftliche Ausbildung in der Regel mit der Promotion - abgeschlossen ist" (DFGVordruck 1.02-8/10). Für Angehörige der Institute und Mitgliedseinrichtungen der Max-Planck-Gesellschaft, der Fraunhofer-Gesellschaft, der Helmholtz-Gemeinschaft oder der Wissenschaftsgemeinschaft Gottfried Wilhelm Leibniz sowie für Angehörige von mit diesen Organisationen assoziierten Forschungseinrichtungen, die aus öffentlichen Mitteln grundfinanziert werden, und für Angehörige deutscher Standorte international getragener Forschungseinrichtungen gelten besondere Bestimmungen (für Details siehe z. B. DFG-Merkblatt 1.02). Möglichkeiten zur Antragstellung gibt es jedoch auch für diesen Personenkreis, insbesondere für Wissenschaftler und Wissenschaftlerinnen mit einem befristeten Arbeitsverhältnis.

Welches Programm bietet sich speziell für Nachwuchswissenschaftlerinnen und -wissenschaftler an? In der frühen Postdoktorandenphase sprechen mehrere Gründe für ein Forschungsstipendium. Zum einen ist der wissenschaftliche Gewinn durch einen Forschungsaufenthalt im Ausland oftmals sehr groß. Zum zweiten ist die Förderquote deutlich höher als bei anderen DFG-Programmen (siehe oben). Zum dritten besteht im Anschluss an den Auslandsaufenthalt die Möglichkeit auf ein Rückkehrstipendium zur Wiedereingliederung in das deutsche Wissenschaftssystem. Die Forschungsstipendien dauern meist zwei Jahre und beinhalten neben dem Stipendium auch Fahrtkostenzuschuss, Auslandszuschlag, Kinderzulage und Unterstützung für Erziehungsleistungen. Für Nachwuchswissenschaftler und Nachwuchswissenschaftlerinnen, die bereits ein eigenes Forschungsprofil nachweisen können (das kann schon kurz nach der Promotion sein, oder aber erst einige Jahre danach), ist ferner ein Antrag auf eine eigene Stelle im Rahmen eines Sachbeihilfe-Projekts möglich. Dabei ist dem Antrag eine Erklärung der aufnehmenden Institution beizufügen, in der sie sich verpflichtet, die Arbeitgeberfunktionen für die Laufzeit der Bewilligung zu übernehmen. Da die bürokratischen Mühlen an Universitäten gelegentlich langsam mahlen, sollte man sich rechtzeitig darum kümmern, einen solchen Antrag zu stellen. Schließlich seien noch das Emmy Noether-Programm und das Heisenberg-Programm erwähnt, die attraktive, aber auch sehr kompetitive Möglichkeiten darstellen, für bis zu fünf Jahre gefördert zu werden. Das Emmy Noether-Programm richtet sich an Postdoktorandinnen und Postdoktoranden mit in der Regel zwei bis vier Jahren Forschungserfahrung (davon mindestens ein Jahr im Ausland) und beinhaltet die Förderung einer Nachwuchsgruppe. Nachwuchswissenschaftlerinnen und -wissenschaftler sollten des Emmy NoetherProgramm in Erwägung ziehen, wenn die genannten Voraussetzungen erfüllt sind und innovative Ideen für ein exzellentes, langjähriges, im Team zu bearbeitendes Forschungsprogramm vorhanden sind. Das Heisenberg-Programm richtet sich an Nachwuchswissenschaftler und Nachwuchswissenschaftlerinnen, die ihre Berufbarkeit auf eine Professur (durch eine Habilitation oder äquivalente Leistungen) nachweisen können und durch besonders herausragende wissenschaftliche Leistungen ausgewiesen sind.

Was zeichnet eigentlich einen guten Antrag aus? Nun, zunächst natürlich eine lohnende wissenschaftliche Fragestellung, verbunden mit guten, innovativen Ideen. Darüber hinaus müssen selbstverständlich die Formalia sorgfältig beachtet werden. Beispielsweise sollte der Antrag keinesfalls mehr als 20 Seiten umfassen. Im Fachkollegium Psychologie haben sich über die letzte Jahre hinweg einige Kostenpauschalen etabliert, die man ebenfalls berücksichtigen sollte (Versuchspersonenstunden $8 €$, Reisekosten $1750 € /$ Stelle/Jahr, Publikationskosten $750 € /$ Jahr). Es sollten keine Mittel für Positionen beantragt werden, die zu einer zeitgemäßen Grundausstattung gehören. In der Psychologie sind das etwa Arbeitsplatzrechner, Festplatten, CDs, grundständige Softwareprogramme (wie SPSS, MatLab usw.), Kopien und ähnliches. Projektspezifische Kosten, die man nicht als Grundausstattung voraussetzen kann, können jedoch beantragt werden. beispielsweise wenn in einem Projekt ungewöhnlich viele Kopien für Befragungen benötigt werden, oder wenn ganz spezielle Hardware (ein Rechner mit besonderen Anforderungen) erforderlich ist. Bei der Kalkulation der Kosten sollte man realistisch bleiben, denn die Kosten müssen nachvollziehbar begründet sein. Es wird weder von den Gutachtern noch vom Kollegium goutiert, wenn man in dieser Hinsicht fehlenden Realitätssinn dokumentiert.

Problematisch sind insbesondere argumentative Schwächen im Antrag. Der Antrag sollte für sich verständlich geschrieben sein und inhaltlich überzeugen. Wenn sich bereits die fachnahen Gutachter mühsam durch den Antrag quälen müssen, wenn die theoretische Einbettung nicht deutlich wird, wenn die Hypothesen und die Variablen nicht klar werden, dann wird der Antrag schwerlich überzeugen können. Überraschend häufig wird leider auch der zu erwartende Erkenntnisfortschritt nicht klar genug herausgearbeitet. Die Thematik muss ferner sinnvoll eingrenzt sein, die Methoden müssen angemessen sein und vom Antragsteller bzw. der Antragstellerin beherrscht werden. Die Durchführbarkeit des Forschungsvorhabens im geplanten Zeitrahmen muss gewährleistet sein. Für die Abschätzung der Realisierbarkeit und der Erfolgsaussichten eines Projekts spielt folglich nicht nur der Antrag selbst, sondern auch die inhaltliche und methodische Qualifikation der Antragstellerin bzw. des Antragstellers in Bezug auf das beantragte Projekt eine nicht zu vernachlässigende Rolle.

Aus unserer Sicht sind die oben erwähnten Mängel oft vermeidbar, also häufig nicht auf eine grundsätzlich fehlende wissenschaftliche Tragfähigkeit des Projekts oder mangelnde Qualifikation der Antragsteller oder Antragstellerinnen zurückzuführen. Es empfiehlt sich daher, fertige Anträge nochmals aus der Perspektive eines Gutachters und einer Fachkollegiatin zu lesen und gegebenenfalls in dieser Hinsicht zu optimieren. Gerade bei Erstanträgen und Anträgen auf eigene Stelle sollte man den Antrag vor Einreichung von einer in DFG-Anträgen erfahrenen Kollegin bzw. einem Kollegen kritisch lesen 
und kommentieren lassen. Soweit zu den guten Ratschlägen, denen wir im Übrigen selbst nicht immer gerecht werden, denn auch unsere eigenen Anträge werden gelegentlich abgelehnt.

Wenn der Antrag abgeschickt ist, braucht es etwa sechs Monate Geduld, bevor man über Förderung oder Ablehnung unterrichtet wird. Das ist im Vergleich zu anderen Förderorganisationen keine lange Bearbeitungszeit. Im Fall einer Bewilligung kann man sich freuen, sollte aber auch hier diverse Formalia und inhaltliche Aspekte beachten. Möglicherweise werden relevante Gutachterhinweise mitgeteilt, die man berücksichtigen sollte (oder nur gut begründet nicht berücksichtigen sollte). Man ist zum verantwortungsvollen Umgang mit den bewilligten Mitteln ebenso aufgefordert wie zur Einhaltung der Regeln guter wissenschaftlicher Praxis. Schließlich ist am Ende ein Abschlussbericht gefordert und es werden gute Forschungsergebnisse erhofft, die in Publikationen mit möglichst hoher Sichtbarkeit resultieren. Hier geht Qualität grundsätzlich vor Quantität. Für die Psychologie bedeutet das, dass man besser in angesehenen internationalen Zeitschriften mit externem Begutachtungssystem publizieren sollte als in Journalen oder Medien, die weniger bzw. gar nicht qualitätskontrolliert sind.

Im Falle eines Ablehnungsbescheids erhaltenen Antragsteller eine Begründung, was zur Ablehnung geführt hat. Ist man nach sorgfältiger Prüfung dieser Gründe der Auffassung, dass man nach Überarbeitung und/oder der Einbeziehung überzeugender Vorarbeiten das Forschungsprojekt nochmals vorlegen möchte, ist das durchaus möglich. Eine Ablehnung bedeutet also nicht, dass das Thema oder das Projekt auf Dauer nicht mehr gefördert werden kann. Nicht selten sind überarbeitete und wiedereingereichte Anträge erfolgreich. Hier kann es sinnvoll sein, sich durch die Geschäftsstelle beraten zu lassen, falls man unsicher ist, ob sich eine Überarbeitung lohnen könnte.

Wir hoffen, mit diesen Informationen und Empfehlungen manche Wissenslücke geschlossen zu haben und zur Entmythologisierung der Entscheidungsfindung in der DFG beigetragen zu haben. Nach unserer Einschätzung handelt es sich um ein transparentes und faires System, in dem relativ hohe Erfolgsaussichten auf Förderung bestehen. Die Beurteilung erfolgt nach wissenschaftlichen
Maßstäben, da ausschließlich die Qualität des Vorhabens und die Qualifikation der Antragstellerin bzw. des Antragstellers zur Durchführung des Vorhabens eine Rolle spielen. Das „Ranking“ der Institution, der die Antragstellenden angehören, spielt keine Rolle, ebenso wenig gibt es „politische“ Vorgaben in Bezug auf bestimmte Themengebiete. Jede gute Forschungsidee hat eine realistische Chance auf Förderung, auch wenn die antragstellende Person oder ihre Institution (noch) kaum bekannt ist. Im Übrigen tragen alle Antragstellenden direkt und indirekt zur Entscheidungsfindung bei: als Antragsteller und Antragstellerinnen, die gute (oder relativ gesehen weniger gute) Anträge stellen, als potenzielle Gutachter und Gutachterinnen, die sich aus dem Kreis der Antragsteller und Antragstellerinnen rekrutieren und möglichst aussagekräftige Gutachten erstellen, auf die sich die Entscheidungsfindung stützt, und als Mitglieder im Fachkollegium, die - so hoffen wir - gut für die Qualitätssicherung sorgen.

Nicht zu vergessen ist, dass Fachkollegiatinnen und -kollegiaten von den potenziellen Antragstellern und Antragstellerinnen gewählt werden. Damit sind wir beim letzten Punkt dieses Beitrags, nämlich den anstehenden DFGWahlen 2011. Wir möchten Sie darum bitten, von Ihrem Wahlrecht Gebrauch zu machen. Warum? Weil es wichtig ist, dass die Forschenden in der DFG gut repräsentiert sind und die Gewählten von einer möglichst großen Wahlgemeinde akzeptiert werden. Von Montag, den 07. November 2011, 14.00 Uhr bis Montag den 05. Dezember 2011, 14.00 Uhr finden die Fachkollegienwahl 2011 statt (näheres unter http://www.dfg.de/dfg_profil/gremien/fachkollegien/fk_wahl2011). Über eine hohe Wahlbeteiligung würden wir uns sehr freuen!

\section{Prof. Dr. Erich Schröger}

Deutsche Forschungsgemeinschaft

BioCog: Cognitive including Biological Psychology

Institut für Psychologie

Universität Leipzig

Seeburgstr. 14-20

04103 Leipzig

E-Mail: schroger@rz.uni-leipzig.de 